\title{
High-Energy String Scattering Amplitudes and Signless Stirling Number Identity
}

\author{
Jen-Chi LEE ${ }^{\dagger}$, Catherine H. YAN ${ }^{\ddagger}$ and $Y i Y A N G^{\dagger}$ \\ $\dagger$ Department of Electrophysics, National Chiao-Tung University, Hsinchu, Taiwan, R.O.C. \\ E-mail: jcclee@cc.nctu.edu.tw,yiyang@mail.nctu.edu.tw \\ $\ddagger$ Department of Mathematics, Texas A $\mathscr{E} M$ University, College Station, TX 7r843, USA \\ E-mail: cyan@math.tamu.edu
}

Received April 23, 2012, in final form July 10, 2012; Published online July 18, 2012

http://dx.doi.org/10.3842/SIGMA.2012.045

\begin{abstract}
We give a complete proof of a set of identities (7) proposed recently from calculation of high-energy string scattering amplitudes. These identities allow one to extract ratios among high-energy string scattering amplitudes in the fixed angle regime from highenergy amplitudes in the Regge regime. The proof is based on a signless Stirling number identity in combinatorial theory. The results are valid for arbitrary real values $L$ rather than only for $L=0,1$ proved previously. The identities for non-integer real value $L$ were recently shown to be realized in high-energy compactified string scattering amplitudes [He S., Lee J.C., Yang Y., arXiv:1012.3158]. The parameter $L$ is related to the mass level of an excited string state and can take non-integer values for Kaluza-Klein modes.
\end{abstract}

Key words: string scattering amplitudes; stirling number identity

2010 Mathematics Subject Classification: 81T30; 83E30

\section{Introduction}

Recently high-energy fixed angle string scattering amplitudes were intensively investigated $[6,7,9]$ for string states at arbitrary mass levels. One of the motivation of this calculation has been to uncover the fundamental hidden stringy spacetime symmetry conjectured more than twenty years ago in $[12,13,14,15,16]$. It was conjectured in late $80 \mathrm{~s}[12,13]$ that there exist linear relations or symmetries among scattering amplitudes in the high energy fixed angle regime, or Gross regime (GR). In the recent calculations, an infinite number of linear relations among high-energy scattering amplitudes of different string states were derived and the complete ratios among the amplitudes at each fixed mass level can be determined. An important new ingredient of this string amplitude calculation was based on an old conjecture of [8, 10, 20, 23, 24, 25] on the decoupling of zero-norm states (ZNS) in the spectrum, in particular, the identification of inter-particle symmetries induced by the inter-particle ZNS [24, 25] in the spectrum.

Another fundamental regime of high-energy string scattering amplitudes is the Regge regime (RR) $[1,2,3,5,27,29]$. See also [4, 11, 19]. It was found [22] that the high-energy string scattering amplitudes in the GR and RR contain information complementary to each other. On the other hand, since the decoupling of ZNS applies to all kinematic regimes, one expects that the ratios obtained from the decoupling of ZNS in the GR are closely related to the decoupling of ZNS or scattering amplitudes in the RR. Moreover, it is conceivable that there exists some link between the patterns of the high-energy scattering amplitudes in the GR and RR. It was found that the number of high-energy scattering amplitudes for each fixed mass level in the $\mathrm{RR}$ is much more numerous than that of GR calculated previously. In contrast to the case of scattering amplitudes in the GR, there is no linear relation among scattering amplitudes in the 
RR. Moreover, it was discovered that the leading order amplitudes at each fixed mass level in the RR can be expressed in terms of the Kummer function of the second kind. More surprisingly, for those leading order string tree four-point high-energy amplitudes $A^{(N, 2 m, q)}$ (see equation (4)) in the RR with the same type of $(N, 2 m, q)$ as those of GR, one can extract from them the ratios $T^{(N, 2 m, q)} / T^{(N, 0,0)}$ (see equations $(1)$ and $\left.(2)\right)$ in the GR by using this Kummer function. The calculation was based on a set of identities equation (7) which depend on an integer parameter $L\left(M_{2}^{2}\right)=1-N$ where $M_{2}^{2}=2(N-1)$ is the mass square of the second string scattering state (here, for simplicity, one chooses the other three string states to be tachyons). The calculation can be done for both the case of open string and the closed string as well.

The proof of these identities for $L=0,1$ was previously given in [17, 22] based on a set of signed Stirling number identities developed in 2007 [21]. However, the proof of these identities for arbitrary integer values $L$ is still lacking, and it is crucial to complete the proof in order to link high-energy string scattering amplitudes in the RR and GR regimes as claimed above. Moreover, it was discovered recently [18] that in order to link the RR and GR string amplitudes for the scattering compactified on tori, one needs to prove the identities for arbitrary real values $L$.

In this letter, we are going to prove these identities for arbitrary real values $L$ by using a signless Stirling number identity. It is remarkable to see that the identities suggested by string theory calculation can be rigorously proved by a totally different mathematical method in combinatorial theory. It is also very interesting to see that, physically, the identities for arbitrary real values $L$ in equation (8) can only be realized in high-energy compactified string scattering amplitudes considered very recently [18]. This is mainly due to the relation $M^{2}=$ $\left(K^{25}\right)^{2}+\hat{M}^{2}$ where $\hat{M}^{2}=2(N-1)$ and $K^{25}=\frac{2 \pi l-\theta_{l}+\theta_{i}}{2 \pi R}$ (see, e.g., [28]) is the generalized KK internal momentum corresponding to the compactified string coordinate [18]. In the definition of $K^{25}, l$ is the quantized momentum, $R$ is the radius of compactified $S^{1}$, and we have included a nontrivial Wilson line with $U(n)$ Chan-Paton factors, $i, l=1,2, \ldots, n$. All other high-energy string scattering amplitudes calculated previously [17,22] correspond to integer values of $L$ only.

\section{GR and RR amplitudes}

We begin with a brief review of the high-energy string scattering in the fixed angle regime,

$$
s,-t \rightarrow \infty, \quad t / s \approx-\sin ^{2} \frac{\phi}{2}=\text { fixed } \quad(\text { but } \phi \neq 0),
$$

where $s, t$ and $u$ are the Mandelstam variables and $\phi$ is the CM scattering angle. It was shown [6] that for the 26D open bosonic string the only states that will survive the high-energy limit at mass level $M_{2}^{2}=2(N-1)$ are of the form (we choose the second state of the four-point function to be the higher spin string state)

$$
|N, 2 m, q\rangle \equiv\left(\alpha_{-1}^{T}\right)^{N-2 m-2 q}\left(\alpha_{-1}^{L}\right)^{2 m}\left(\alpha_{-2}^{L}\right)^{q}|0, k\rangle,
$$

where the polarizations of the 2 nd particle with momentum $k_{2}$ on the scattering plane were defined to be $e^{P}=\frac{1}{M_{2}}\left(E_{2}, k_{2}, 0\right)=\frac{k_{2}}{M_{2}}$ as the momentum polarization, $e^{L}=\frac{1}{M_{2}}\left(k_{2}, E_{2}, 0\right)$ the longitudinal polarization and $e^{T}=(0,0,1)$ the transverse polarization which lies on the scattering plane. The three vectors $e^{P}, e^{L}$ and $e^{T}$ satisfy the completeness relation [7] $\eta_{\mu \nu}=$ $\sum_{\alpha, \beta} e_{\mu}^{\alpha} e_{\nu}^{\beta} \eta_{\alpha \beta}$ where $\mu, \nu=0,1,2$ and $\alpha, \beta=P, L, T$, and $\alpha_{-1}^{T}=\sum_{\mu} e_{\mu}^{T} \alpha_{-1}^{\mu}, \alpha_{-1}^{T} \alpha_{-2}^{L}=$ $\sum_{\mu} e_{\mu}^{T} e_{\nu}^{L} \alpha_{-1}^{\mu} \alpha_{-2}^{\nu}$ etc. $\operatorname{diag} \eta_{\mu \nu}=(-1,1,1)$. In equation $(1), N, m$ and $q$ are non-negative integers and $N \geq 2 m+2 q$. These integers characterise the mass square and "spin" of the higher string states. Note that $e^{P}$ approaches to $e^{L}$ in the GR [7] or equivalently the $e^{P}$ polarizations can be gauged away using ZNS. So we did not put $e^{P}$ components in equation (1). For simplicity, we choose the particles associated with momenta $k_{1}, k_{3}$ and $k_{4}$ to be tachyons. It 
turned out that the high-energy fixed angle scattering amplitudes can be calculated by using the saddle-point method [6]. An infinite number of linear relations among four-point high-energy scattering amplitudes of different string states were derived and the complete ratios among the amplitudes at each fixed mass level can be calculated to be [6]

$$
\frac{T^{(N, 2 m, q)}}{T^{(N, 0,0)}}=\left(-\frac{1}{M_{2}}\right)^{2 m+q}\left(\frac{1}{2}\right)^{m+q}(2 m-1) ! ! .
$$

Alternatively, the ratios can be calculated by the method of decoupling of two types of ZNS in the old covariant first quantized string spectrum. Since the decoupling of ZNS applies to all string loop order, the ratios calculated in equation (2) are valid to all string loop order. Similarly, the ratios for closed string, superstring and D-brane scattering amplitudes [9] can be obtained.

Another high-energy regime of string scattering amplitudes, which contains complementary information of the theory, is the fixed momentum transfer $t$ or RR. That is in the kinematic regime

$$
s \rightarrow \infty, \quad \sqrt{-t}=\text { fixed } \quad(\text { but } \sqrt{-t} \neq \infty) .
$$

It was found [22] that the number of high-energy scattering amplitudes for each fixed mass level in this regime is much more numerous than that of fixed angle regime calculated previously. On the other hand, it seems that both the saddle-point method and the method of decoupling of zero-norm states adopted in the calculation of fixed angle regime do not apply to the case of Regge regime. However the calculation is still manageable, and the general formula for the high energy $(s, t)$ channel open string scattering amplitudes at each fixed mass level can be written down explicitly.

It was shown that a class of high-energy open string states in the Regge regime at each fixed mass level $N=\sum_{l, m} l p_{l}+m q_{m}$ are $[17,22]$

$$
\left|p_{l}, q_{m}\right\rangle=\prod_{l>0}\left(\alpha_{-l}^{T}\right)^{p_{l}} \prod_{m>0}\left(\alpha_{-m}^{L}\right)^{q_{m}}|0, k\rangle
$$

As explained in [17] for the purpose of connecting the RR with the GR limit of a scattering amplitude, suffices to consider scattering amplitudes involving only the vertex in equation (3). The complete high energy vertex can be found in [17]. The momenta of the four particles on the scattering plane are

$$
\begin{array}{ll}
k_{1}=\left(+\sqrt{p^{2}+M_{1}^{2}},-p, 0\right), & k_{2}=\left(+\sqrt{p^{2}+M_{2}^{2}},+p, 0\right), \\
k_{3}=\left(-\sqrt{q^{2}+M_{3}^{2}},-q \cos \phi,-q \sin \phi\right), & k_{4}=\left(-\sqrt{q^{2}+M_{4}^{2}},+q \cos \phi,+q \sin \phi\right),
\end{array}
$$

where $p \equiv|\vec{p}|, q \equiv|\vec{q}|$ and $k_{i}^{2}=-M_{i}^{2}$. The relevant kinematics are

$$
\begin{aligned}
e^{P} \cdot k_{1} \simeq-\frac{s}{2 M_{2}}, & e^{P} \cdot k_{3} \simeq-\frac{\tilde{t}}{2 M_{2}}=-\frac{t-M_{2}^{2}-M_{3}^{2}}{2 M_{2}}, \\
e^{L} \cdot k_{1} \simeq-\frac{s}{2 M_{2}}, & e^{L} \cdot k_{3} \simeq-\frac{\tilde{t}^{\prime}}{2 M_{2}}=-\frac{t+M_{2}^{2}-M_{3}^{2}}{2 M_{2}},
\end{aligned}
$$

and

$$
e^{T} \cdot k_{1}=0, \quad e^{T} \cdot k_{3} \simeq-\sqrt{-t},
$$


where $\tilde{t}$ and $\tilde{t}^{\prime}$ are related to $t$ by finite mass square terms

$$
\tilde{t}=t-M_{2}^{2}-M_{3}^{2}, \quad \tilde{t}^{\prime}=t+M_{2}^{2}-M_{3}^{2} .
$$

Note that $e^{P}$ does not approach to $e^{L}$ in the RR. The Regge scattering amplitude for the $(s, t)$ channel was calculated to be [22] (we choose to calculate $e^{L}$ amplitudes, the $e^{P}$ amplitudes can be similarly discussed)

$$
A^{(N, 2 m, q)}(s, t)=s^{\alpha(t)} \sqrt{-t}^{N-2 m-2 q}\left(\frac{1}{2 M_{2}}\right)^{2 m+q} \cdot 2^{2 m}\left(\tilde{t}^{\prime}\right)^{q} U\left(-2 m, \frac{t}{2}+2-2 m, \frac{\tilde{t}^{\prime}}{2}\right)
$$

where the level independent [22] exponent $\alpha(t)=a(0)+\alpha^{\prime} t, a(0)=1$ and $\alpha^{\prime}=\frac{1}{2}$. In equation (4) $U$ is the Kummer function of the second kind and is defined to be

$$
U(a, c, x)=\frac{\pi}{\sin \pi c}\left[\frac{M(a, c, x)}{(a-c) !(c-1) !}-\frac{x^{1-c} M(a+1-c, 2-c, x)}{(a-1) !(1-c) !}\right], \quad c \neq 2,3,4, \ldots,
$$

where $M(a, c, x)=\sum_{j=0}^{\infty} \frac{(a)_{j}}{(c)_{j}} \frac{x^{j}}{j !}$ is the Kummer function of the first kind. Note that the second argument of Kummer function $c=\frac{t}{2}+2-2 m$ is a function of the variable $t$, and is not a constant as it was in the literature previously.

It can be seen from equation (4) that the Regge scattering amplitudes at each fixed mass level are no longer proportional to each other. The ratios are $t$ dependent functions and can be calculated to be [22]

$$
\begin{aligned}
\frac{A^{(N, 2 m, q)}(s, t)}{A^{(N, 0,0)}(s, t)}= & (-1)^{m}\left(-\frac{1}{2 M_{2}}\right)^{2 m+q}\left(\tilde{t}^{\prime}-2 N\right)^{-m-q}\left(\tilde{t}^{\prime}\right)^{2 m+q} \\
& \times \sum_{j=0}^{2 m}(-2 m)_{j}\left(-1+N-\frac{\tilde{t}^{\prime}}{2}\right)_{j} \frac{\left(-2 / \tilde{t}^{\prime}\right)^{j}}{j !}+O\left\{\left(\frac{1}{\tilde{t}^{\prime}}\right)^{m+1}\right\},
\end{aligned}
$$

where $(x)_{j}=x(x+1)(x+2) \cdots(x+j-1)$ is the Pochhammer symbol which can be expressed in terms of the signed Stirling number of the first kind $s(n, k)$ as following

$$
(x)_{n}=\sum_{k=0}^{n}(-)^{n-k} s(n, k) x^{k}
$$

It was proposed in [22] that the coefficients of the leading power of $\tilde{t}^{\prime}$ in equation (5) can be identified with the ratios in equations (2). To ensure this identification

$$
\lim _{\tilde{t}^{\prime} \rightarrow \infty} \frac{A^{(N, 2 m, q)}}{A^{(N, 0,0,)}}=\frac{T^{(N, 2 m, q)}}{T^{(N, 0,0)}}=\left(-\frac{1}{M_{2}}\right)^{2 m+q}\left(\frac{1}{2}\right)^{m+q}(2 m-1) ! !,
$$

one needs the following identity

$$
\begin{aligned}
& \sum_{j=0}^{2 m}(-2 m)_{j}\left(-L-\frac{\tilde{t}^{\prime}}{2}\right)_{j} \frac{\left(-2 / \tilde{t}^{\prime}\right)^{j}}{j !} \\
& \quad=0 \cdot\left(-\tilde{t}^{\prime}\right)^{0}+0 \cdot\left(-\tilde{t}^{\prime}\right)^{-1}+\cdots+0 \cdot\left(-\tilde{t}^{\prime}\right)^{-m+1}+\frac{(2 m) !}{m !}\left(-\tilde{t}^{\prime}\right)^{-m}+O\left\{\left(\frac{1}{\tilde{t}^{\prime}}\right)^{m+1}\right\},
\end{aligned}
$$

where $L=1-N$ and is an integer. Similar identification can be extended to the case of closed string as well. For all four classes of high-energy superstring scattering amplitudes, $L$ is an 
integer too [17]. A recent work on string D-particle scattering amplitudes [26] also gives an integer value of $L$. Note that $L$ affects only the sub-leading terms in $O\left\{\left(\frac{1}{\tilde{t}^{\prime}}\right)^{m+1}\right\}$. Here we give a simple example for $m=3$ [17]

$$
\begin{aligned}
\sum_{j=0}^{6}(-2 m)_{j}\left(-L-\frac{\tilde{t}^{\prime}}{2}\right)_{j} \frac{\left(-2 / \tilde{t}^{\prime}\right)^{j}}{j !} & \\
= & \frac{120}{\left(-\tilde{t}^{\prime}\right)^{3}}+\frac{720 L^{2}-2640 L+2080}{\left(-\tilde{t}^{\prime}\right)^{4}}+\frac{480 L^{4}-4160 L^{3}+12000 L^{2}-12928 L+3840}{\left(-\tilde{t}^{\prime}\right)^{5}} \\
& +\frac{64 L^{6}-960 L^{5}+5440 L^{4}-14400 L^{3}+17536 L^{2}-7680 L}{\left(-\tilde{t}^{\prime}\right)^{6}} .
\end{aligned}
$$

Mathematically, equation (7) was exactly proved [17,22] for $L=0,1$ by a calculation based on a set of signed Stirling number identities developed very recently in combinatorial theory in [21]. For general integer $L$ cases, only the identity corresponding to the nontrivial leading term $\frac{(2 m) !}{m !}\left(-\tilde{t}^{\prime}\right)^{-m}$ was rigorously proved [17], but not for other "0 identities". A numerical proof of equation (7) was given in [17] for arbitrary real values $L$ and for non-negative integer $m$ up to $m=10$. It was then conjectured that [17] equation (7) is valid for any real number $L$ and any non-negative integer $m$. It is important to prove equation (7) for any non-negative integer $m$ and arbitrary real values $L$, since these values can be realized in the high-energy scattering of compactified string states, as was shown recently in [18]. Real values of $L$ appear in string compactifications due to the dependence on the generalized KK internal momenta $K_{i}^{25}$ [18]

$$
L=1-N-\left(K_{2}^{25}\right)^{2}+K_{2}^{25} K_{3}^{25} .
$$

All other high-energy string scattering amplitudes calculated previously [17, 22] correspond to integer value of $L$ only. It is thus of importance to rigorously prove the validity of equation (7) for arbitrary real values $L$.

\section{Proof of the identity}

We now proceed to prove equation (7). We first rewrite the left-hand side of equation (7) in the following form

$$
\begin{aligned}
\sum_{j=0}^{2 m}( & -2 m)_{j}\left(-L-\frac{\tilde{t}^{\prime}}{2}\right)_{j} \frac{\left(-2 / \tilde{t}^{\prime}\right)^{j}}{j !} \\
& =\sum_{j=0}^{2 m}(-1)^{j}\left(\begin{array}{c}
2 m \\
j
\end{array}\right) \sum_{l=0}^{j}\left(\begin{array}{l}
j \\
l
\end{array}\right)(-L)_{j-l} \sum_{s=0}^{l} c(l, s)\left(-\frac{2}{\tilde{t}^{\prime}}\right)^{j-s},
\end{aligned}
$$

where we have used the identity $(a+b)_{j}=\sum_{l=0}^{j}\left(\begin{array}{l}j \\ l\end{array}\right)(a)_{j-l}(b)_{l}$ and have introduced the signless Stirling number of the first kind $c(l, s)$ to expand the Pochhammer symbol

$$
(x)_{n}=\sum_{k=0}^{n} c(n, k) x^{k} .
$$

The coefficient of $\left(-2 / \tilde{t}^{\prime}\right)^{i}$ in equation (9), which will be defined as $G(m, i)$, can be read off from the equation as

$$
G(m, i)=\sum_{j=0}^{2 m} \sum_{l=0}^{j}(-1)^{j+i}\left(\begin{array}{c}
2 m \\
j
\end{array}\right)\left(\begin{array}{l}
j \\
l
\end{array}\right)(-L)_{j-l} c(l, j-i) .
$$


One needs to prove that

1. $G(m, m)=(2 m-1) !$ !, for all $L \in \mathbb{R}$;

2. $G(m, i)=0$, for all $L \in \mathbb{R}$ and $0 \leq i<m$.

From the definition of $c(n, k)$ in (10), we note that $c(n, k) \neq 0$ only if $0 \leq k \leq n$. Thus $c(l, j-i) \neq 0$ only if $j \geq i$ and $l \geq j-i$. We can rewrite $G(m, i)$ as

$$
\begin{aligned}
G(m, i) & =\sum_{j=i}^{2 m} \sum_{l=j-i}^{j}(-1)^{j}\left(\begin{array}{c}
2 m \\
j
\end{array}\right)\left(\begin{array}{l}
j \\
l
\end{array}\right)(-L)_{j-l} c(l, j-i) \\
& =\sum_{k=0}^{2 m-i} \sum_{p=0}^{i}(-1)^{k+i}\left(\begin{array}{c}
2 m \\
i+k
\end{array}\right)\left(\begin{array}{c}
i+k \\
p+k
\end{array}\right)(-L)_{i-p} c(k+p, k) \\
& =(-1)^{i} \sum_{p=0}^{i}(-L)_{i-p}\left(\begin{array}{c}
2 m \\
i-p
\end{array}\right) \sum_{k=0}^{2 m-i}(-1)^{k}\left(\begin{array}{c}
2 m-i+p \\
k+p
\end{array}\right) c(k+p, k) \\
& \equiv(-1)^{i} \sum_{p=0}^{i}(-L)_{i-p}\left(\begin{array}{c}
2 m \\
i-p
\end{array}\right) S_{2 m-i}(p),
\end{aligned}
$$

where we have defined

$$
S_{N}(p)=\sum_{k=0}^{N}(-1)^{k}\left(\begin{array}{c}
N+p \\
k+p
\end{array}\right) c(k+p, k) .
$$

It is easy to see that for fixed $m$ and $0 \leq i<m, G(m, i)$ is a polynomial of $L$ of degree $i$, expanded with the basis $1,(-L)_{1},(-L)_{2}, \ldots$ Note that $p \leq i<m$, so $2 m-i \geq p+1$. For equation (12), we want to show that $S_{N}(p)=0$ for $N \geq p+1$. For this purpose, we define the functions

$$
C_{n}(x)=\sum_{k \geq 0} c(k+n, k) x^{k+n} .
$$

The recurrence of the signless Stirling number identity

$$
c(k+n, k)=(n+k-1) c(n+k-1, k)+c(n+k-1, k-1)
$$

leads to the equation

$$
C_{n}(x)=\frac{x^{2}}{1-x} \frac{d}{d x} C_{n-1}(x),
$$

with the initial value

$$
C_{0}(x)=\frac{1}{1-x} .
$$

The first couple of $C_{n}(x)$ can be calculated to be

$$
C_{1}(x)=\frac{x^{2}}{(1-x)^{3}}, \quad C_{2}(x)=\frac{x^{4}+2 x^{3}}{(1-x)^{5}}, \quad C_{3}(x)=\frac{x^{6}+8 x^{5}+6 x^{4}}{(1-x)^{7}} .
$$

Now by induction, it is easy to show that

$$
\begin{aligned}
& C_{n}(x)=\frac{f_{n}(x)}{(1-x)^{2 n+1}}, \quad \text { where } \quad f_{n}(x)=x^{2 n}+\mathcal{O}\left(x^{2 n-1}\right), \\
& f_{n}(1)=(2 n-1) ! !
\end{aligned}
$$




\section{Proof. If}

$$
f_{n}(1)=(2 n-1) ! !
$$

then

$$
f_{n+1}(x)=(1-x)^{2 n+3} \frac{x^{2}}{1-x} \frac{d}{d x}\left[\frac{f_{n}(x)}{(1-x)^{2 n+1}}\right]=x^{2}\left[(2 n+1) f_{n}(x)+(1-x) f_{n}^{\prime}(x)\right]
$$

when $x=1$, since $f_{n}(x)$ is a polynomial, this gives

$$
f_{n+1}(1)=(2 n+1) f_{n}(1)=(2 n+1) ! !=[2(n+1)-1] ! ! .
$$

In order to prove equation (12), we note that

$$
(-1)^{N} S_{N}(p)=\sum_{k=0}^{N}(-1)^{N+k}\left(\begin{array}{c}
N+p \\
k+p
\end{array}\right) c(k+p, k)
$$

is the coefficient of $x^{N+p}$ in the function

$$
\begin{aligned}
(1-x)^{N+p} C_{p}(x) & =(1-x)^{N+p} \sum_{k \geq 0} c(k+p, k) x^{k+p} \\
& =\sum_{k \geq 0} \sum_{m=0}^{N+p}\left(\begin{array}{c}
N+p \\
m
\end{array}\right)(-1)^{N+p-m} c(k+p, k) x^{N+2 p+k-m} .
\end{aligned}
$$

On the other hand, the above function is a polynomial when $N>p$ due to equation (15)

$$
(1-x)^{N+p} C_{p}(x)=f_{p}(x)(1-x)^{N-p-1}=x^{N+p-1}+\mathcal{O}(\cdots) .
$$

It is obvious that the coefficient of $x^{N+p}$ in equation (17) is zero. This proves that $S_{N}(p)=0$ for $N>p$. For $m>i \geq 0$ and $0 \leq p \leq i, 2 m-i>i \geq p$, we have $S_{2 m-i}(p)=0$ and thus equation (12),

$$
G(m, i)=(-1)^{i} \sum_{p=0}^{i}(-L)_{i-p}\left(\begin{array}{c}
2 m \\
i-p
\end{array}\right) S_{2 m-i}(p)=0 .
$$

In order to prove the first identity in equation (11), we first note that the above argument remains true for $i=m$ and $0 \leq p<i$, i.e. $2 m-i=i>p$ and thus $S_{2 m-i}(p)=0$. So $G(m, m)$ only receives contributions from the term with $p=i=m$. By using equation (13) and (14), we can evaluate

$$
G(m, m)=(-1)^{m} S_{m}(m)=\sum_{k=0}^{m}(-1)^{k+m}\left(\begin{array}{c}
2 m \\
k+m
\end{array}\right) c(k+p, k) .
$$

Equation (18) corresponds to the coefficient of $x^{2 m}$ in the function

$$
(1-x)^{2 m} C_{m}(x)=\frac{f_{m}(x)}{1-x}=f_{m}(x)\left(1+x+x^{2}+\cdots\right) .
$$

By equation (16), this coefficient is

$$
f_{m}(1)=(2 m-1) ! !
$$

This proves equation (11). We thus have completed the proof of equation (7) for any non-negative integer $m$ and any real value $L$. 


\section{Acknowledgments}

We thank Rong-Shing Chang, Song He, Yoshihiro Mitsuka and Keijiro Takahashi for helpful discussions. This work is supported in part by the National Science Council, 50 billions project of Ministry of Education and National Center for Theoretical Science, Taiwan.

\section{References}

[1] Amati D., Ciafaloni M., Veneziano G., Can spacetime be probed below the string size?, Phys. Lett. B 216 (1989), 41-47.

[2] Amati D., Ciafaloni M., Veneziano G., Classical and quantum gravity effects from Planckian energy superstring collisions, Internat. J. Modern Phys. A 3 (1988), 1615-1661.

[3] Amati D., Ciafaloni M., Veneziano G., Superstring collisions at Planckian energies, Phys. Lett. B 197 (1987), $81-88$.

[4] Andreev O., Remarks on the high-energy behavior of string scattering amplitudes in warped spacetimes. II, Phys. Rev. D 71 (2005), 066006, 6 pages, hep-th/0408158.

[5] Brower R.C., Polchinski J., Strassler M.J., Tan C.I., The pomeron and gauge/string duality, J. High Energy Phys. 2007 (2007), no. 12, 005, 62 pages, hep-th/0603115.

[6] Chan C.T., Ho P.M., Lee J.C., Teraguchi S., Yang Y., High-energy zero-norm states and symmetries of string theory, Phys. Rev. Lett. 96 (2006), 171601, 4 pages, hep-th/0505035.

[7] Chan C.T., Lee J.C., Zero-norm states and high-energy symmetries of string theory, Nuclear Phys. B 690 (2004), 3-20, hep-th/0401133.

[8] Chan C.T., Lee J.C., Yang Y., Anatomy of zero-norm states in string theory, Phys. Rev. D 71 (2005), 086005, 14 pages, hep-th/0501020.

[9] Chan C.T., Lee J.C., Yang Y., Scatterings of massive string states from D-brane and their linear relations at high energies, Nuclear Phys. B 764 (2007), 1-14, hep-th/0610062.

[10] Chung T.D., Lee J.C., Discrete gauge states and $w_{\infty}$ charges in $c=12$ D gravity, Phys. Lett. B 350 (1995), 22-27, hep-th/9412095.

[11] Danilov G.S., Lipatov L.N., BFKL pomeron in string models, Nuclear Phys. B 754 (2006), 187-232, hep-ph/0603073.

[12] Gross D.J., High-energy symmetries of string theory, Phys. Rev. Lett. 60 (1988), 1229-1232.

[13] Gross D.J., Strings at super-Planckian energies: in search of the string symmetry. Physics and mathematics of strings, Philos. Trans. Roy. Soc. London Ser. A 329 (1989), 401-413.

[14] Gross D.J., Mañes J.L., The high energy behavior of open string scattering, Nuclear Phys. B 326 (1989), 73-107.

[15] Gross D.J., Mende P.F., String theory beyond the Planck scale, Nuclear Phys. B 303 (1988), $407-454$.

[16] Gross D.J., Mende P.F., The high-energy behavior of string scattering amplitudes, Phys. Lett. B 197 (1987), 129-134.

[17] He S., Lee J.C., Takahashi K., Yang Y., Massive superstring scatterings in the Regge regime, Phys. Rev. D 83 (2011), 066016, 21 pages, arXiv:1001.5392.

[18] He S., Lee J.C., Yang Y., Exponential fall-off behavior of Regge scatterings in compactified open string theory, arXiv:1012.3158.

[19] Kachelriess M., Plumacher M., Remarks on the high-energy behaviour of cross-sections in weak-scale string theories, hep-ph/0109184.

[20] Kao H.C., Lee J.C., Decoupling of degenerate positive-norm states in Witten's string field theory, Phys. Rev. D 67 (2003), 086003, 9 pages, hep-th/0212196.

[21] Kauers M., Summation algorithms for Stirling number identities, J. Symbolic Comput. 42 (2007), 948-970.

[22] Ko S.L., Lee J.C., Yang Y., Patterns of high energy massive string scatterings in the Regge regime, J. High Energy Phys. 2009 (2009), no. 6, 028, 21 pages, arXiv:0812.4190.

[23] Lee J.C., Calculation of zero-norm states and reduction of stringy scattering amplitudes, Progr. Theoret. Phys. 114 (2005), 259-273, hep-th/0302123. 
[24] Lee J.C., Decoupling of degenerate positive-norm states in string theory, Phys. Rev. Lett. 64 (1990), 16361638.

[25] Lee J.C., New symmetries of higher spin states in string theory, Phys. Lett. B 241 (1990), 336-342.

[26] Lee J.C., Mitsuka Y., Yang Y., States scattered from D-particle in the Regge regime and factorized ratios of fixed angle scatterings, Progr. Theoret. Phys. 126 (2011), 397-417, arXiv:1101.1228.

[27] Muzinich I.J., Soldate M., High-energy unitarity of gravitation and strings, Phys. Rev. D 37 (1988), 359-367.

[28] Polchinski J., String theory. Vol. I. An introduction to the bosonic string, Cambridge Monographs on Mathematical Physics, Cambridge University Press, Cambridge, 2005.

[29] Soldate M., Partial-wave unitarity and closed-string amplitudes, Phys. Lett. B 186 (1987), 321-327. 